\title{
Daniel, Ute: Beziehungsgeschichten. Politik und Medien im 20. Jahrhundert, 464 S., Hamburger Edition, Hamburg 2018.
}

\author{
Jörg Requate \\ Online publiziert: 7. Mai 2020 \\ (C) Der/die Autor(en) 2020
}

Er brauche zum Regieren nur „Bild, BamS und die Glotze“, so ein oft kolportiertes, wenngleich nie bestätigtes Zitat vom vermeintlichen Medienkanzler Gerhard Schröder. Die Lektüre von Ute Daniels Buch über das Verhältnis von Politik und Medien hätte ihn möglicherweise eines Besseren belehren und ihm deutlich machen können, dass die Dinge dann doch etwas komplizierter liegen. Denn, so eine zentrale Botschaft des Buches, Allmachtsfantasien erscheinen in dem komplexen Beziehungsgewirr von Politik und Medien in der einen wie in der anderen Richtung fehl am Platz. Vielmehr sei es notwendig, jeweils genau hinzuschauen, wie sich das Verhältnis zwischen Politik und Medien beziehungsweise den jeweils handelnden Akteurinnen und Akteuren gestaltete. Es sei bislang niemandem gelungen, so Daniel in ihrer Einleitung, die Ausgestaltung der Beziehungen zwischen Politik und Medien „von den strukturellen Faktoren“ (S. 12) - also dem Typus der Mediensysteme, den parteipolitischen Unterschieden et cetera - her zu erklären und, so die Überzeugung der Autorin, dies werde wohl auch nie gelingen. Die Aussage, dass Politik und Medien einander bräuchten, sei zwar richtig, aber letztlich unbefriedigend. Maßgeblich für sie sei vielmehr, ,unter welchen Bedingungen die beiden Akteursgruppen sich in welchen Formen wechselseitig brauchen, unter welchen Bedingungen sich also diese wechselseitige Abhängigkeit in kongenialen Formen der Kooperation, Vertrautheit und Aktionsgemeinschaft äußert" (S. 13). Einen übergreifenden Erklärungsansatz strebt Daniel dabei genauso wenig an wie den Entwurf einer Entwicklungstendenz. Gleichwohl lässt sich ein gewisser Grundtenor erkennen: Die Autorin sieht ohne Zweifel in der Pressefreiheit ein zentrales, aber immer wieder bedrohtes Gut und ist zudem gleichsam leitmotivisch um eine gewisse Demystifizierung allzu einfacher Vorstellungen von Instrumentalisierungen der Presse einerseits oder deren Heroisierungen andererseits bemüht.

Dazu sucht sich Daniel markante historische Konstellationen in einer Art deutschenglischen Parallelgeschichte heraus und unterzieht diese einer vergleichenden Analyse. Die einzige Ausnahme bildet das dritte Kapitel, das sich Goebbels' Sportpalastrede vom 18. Februar 1943 und dessen Nachwirkungen widmet. Die gänzlich

J. Requate $(\triangle)$

Universität Kassel, Kassel, Deutschland

E-Mail: joerg.requate@ uni-kassel.de 
unterschiedlichen politischen Kontexte machten hier einen Vergleich unmöglich, der für die anderen Kapitel dagegen grundlegend ist.

Das erste Kapitel widmet sich dem Verhältnis von Medien und Politik im Ersten Weltkrieg. In beiden Ländern gab es bei Regierungen und Militär sehr weitreichende Vorstellungen darüber, wie die Presse propagandistisch zu nutzen sei. Daniel setzt hier jedoch gleich einen Kontrapunkt und versucht dem Bild einer durchweg kontrollierten und gelenkten Presse entgegenzuwirken. Tatsächlich gab es in Großbritannien nur eine sehr zurückhaltende, im Wesentlichen auf militärisch relevante Informationen beschränkte Zensur, wohl aber eine sehr wirksame Selbstzensur. Statt in einen Gegensatz zu geraten, habe sich das ,,Vertraulichkeitskartell“ - ein Schlüsselbegriff für Ute Daniels Darstellung insgesamt - von Politikern und Journalisten noch weiter vertieft. Politische Fragen und sogar militärische Belange seien weiter in der Presse verhandelbar gewesen, aber gleichsam in einem ,,verantwortlichen Rahmen“ (sprich: durch eine sehr restriktive Weitergabe von Informationen), wodurch die Bedeutung der Presse als Mitspieler auf dem Feld der Politik noch einmal gesteigert worden sei. Der Unterschied zu Deutschland war grundsätzlich eklatant: Die Zensur war hier allgegenwärtig, doch sei die Vielzahl der Zensurstellen nicht unbedingt als „Gradmesser für Ausmaß und Effizienz der Presselenkung“ (S. 91) zu werten. Daniel hat sicher recht, wenn sie die Zensur als chaotisch beschreibt und die zum Teil kontraproduktiven Effekte der Propaganda hervorhebt. Allerdings betont sie auch, dass ,gegen Ende des Krieges [...] die Journalisten hier [gemeint waren die Pressekonferenzen, J. R.] praktisch gar nichts mehr [erfuhren, J. R.], was zur Veröffentlichung bestimmt war“ (S. 94). Der „Gradmesser“ von Wirksamkeit ist deshalb nicht immer eindeutig bestimmbar.

Das zweite Kapitel befasst sich mit der politischen Bedeutung der sogenannten „Pressezaren“ in der Zwischenkriegszeit. Der Konzentrationsprozess der Presse und der weitere Aufstieg der Massenpresse hatten sowohl in Deutschland als auch in England dazu geführt, dass mit einigen wenigen Verlegern eine enorme publizistische Macht assoziiert wurde. In England war dies allen voran Lord Northcliffe, in Deutschland Alfred Hugenberg. Im Einzelnen sehr unterschiedlich von ihrer ganzen unternehmerischen und publizistischen Herangehensweise, war beiden gemeinsam, dass sie gezielt nach politischem Einfluss suchten und diesen sicher auch ausübten. Grundsätzlich argumentiert Daniel zu Recht, dass mit den „Pressezaren“ zwar neue „Player“ in die politische Arena traten, diese aber mit ihren politischen Ansprüchen und mit der Vorstellung, die publizistische in politische Macht ummünzen zu können, weitgehend scheiterten. Allerdings erstaunt in diesem Kapitel etwas, wie ausführlich sich die Autorin mit den jeweiligen politischen Ränkespielen in den betroffenen Parteien Englands und Deutschlands befasst. War es tatsächlich die „Anrüchigkeit“, die der Massenpresse in Deutschland anhaftete, ,die Hugenbergs Rolle in der DNVP jahrelang beschränkte“ (S. 178)? Die Frage, ob oder inwiefern es Hugenberg möglicherweise gelang, publizistische in politische Macht umzusetzen oder warum dies möglicherweise überhaupt nicht funktionierte, scheint auf diese Weise noch weitgehend offen.

Auch in dem sehr knapp gehaltenen Kapitel zur Propaganda im ,Dritten Reich“ geht es der Autorin darum, die vermeintliche Allmacht der Propaganda in Diktaturen zu demystifizieren. Sie konzentriert sich auf die berüchtigte, von Joseph Goebbels 
gehaltene Sportpalastrede, in der es ihm gelang, Jubelstürme für den „totalen Krieg“ auszulösen. Daniel zeigt, wie Goebbels die Rede als ein Medienereignis inszenierte, differenziert zwischen verschiedenen Adressatengruppen und betont, dass die Rede keineswegs ein solcher Propagandaerfolg war, wie sie im Rückblick oft erschien. In der Tat hatte die Rede eine ganze Reihe von unintendierten Folgen - etwa als Frauen aus der Arbeiterschaft ihren Arbeitseinsatz davon abhängig machten, dass auch Frauen ,aus der feinen Gesellschaft“ ihren Beitrag leisteten. So wird man sich gewiss davor hüten müssen, allzu simpel davon auszugehen, dass große Propagandainszenierungen auch entsprechenden Erfolg zeitigten - wie verbreitet die nicht intendierten Folgen waren, ist gleichwohl ebenfalls schwer messbar.

Das vierte Kapitel thematisiert anhand des Profumo-Falls und der Spiegel-Affäre die Rolle der Medien bei der Aufdeckung von Skandalen. Auch an dieser Stelle geht es der Autorin darum, gängige Lesarten zu relativieren: Sie betont hier weniger die aufdeckende Rolle der Medien als die politischen Maßnahmen gegen die Presse, die vor allem im Kontext der Profumo-Affäre zu einer deutlichen Zurückhaltung der Medien geführt hätten. Beide Fälle dienen der Autorin dazu, aufzuzeigen, wie fragil die Pressefreiheit auch in demokratischen Gesellschaften ist. Das letzte Kapitel widmet sich schließlich dem öffentlich-rechtlichen Rundfunk in beiden Ländern. Auch hier verdeutlicht Daniel vor allem, in welchem Maße Politiker strukturellen Einfluss auf die Sender nahmen.

In ihrem Fazit arbeitet die Autorin zwei Grundkonstanten heraus, die in ihren Augen für beide untersuchten Länder zentral waren: das schon genannte Vertraulichkeitskartell, das Politiker und Journalisten gleichsam strukturell miteinander verbindet einerseits sowie die „,deutlich stärkere Stellung der politischen Akteure“ gegenüber den Medien andererseits. Es seien die Regierungen und Parlamente, die die rechtlichen Rahmenbedingungen setzten und die letztlich auch über das Ausmaß oder die Einschränkung der Pressefreiheit entschieden. Daniel setzt hier einen expliziten Kontrapunkt gegen jene, die eine „Übermacht der Medien“ und Demokratien auf dem Weg zu einer Mediokratie sehen. Ihr Plädoyer, stattdessen strikt kontextbezogen das jeweilige Verhältnis von Politik und Medien zu untersuchen, ist wohlbegründet und gut nachvollziehbar. Ihre Analysen belegen den Wert dieses Ansatzes.

Allerdings ist zum einen auffällig, dass sich die Autorin in ihren Untersuchungen jeweils weit mehr mit der politischen als mit der medialen Seite befasst. Die Tatsache, dass Politiker Vorstellungen darüber entwickelten, wie sie über die Änderung der Staatsverträge missliebige Berichterstattung verhindern konnten, sagt noch wenig über tatsächliche Einflussnahme aus. Auch ohne die Rolle der Presse zu heroisieren, ließe sich die Kontrollfunktion der Medien in bestimmten Konstellationen sicher klarer herausarbeiten. Die Frage, in welchem Maße sich Politikerinnen und Politiker den medialen Eigengesetzlichkeiten unterwerfen mussten, wäre es ebenfalls wert, an konkreten Konstellationen zu untersuchen. Doch Daniel betont ausdrücklich, dass es ihr vielmehr um bestimmte Tiefenbohrungen geht, deren Ergebnis eben auch davon abhängt, wo man die Bohrung ansetzt. Mit vielen ihrer Analysen reibt sich das Buch an vorherrschenden Perspektiven und liefert auf diese Weise Reibungsflächen für weitere Forschungen. Im Verhältnis von Politik und Medien ist, wie Ute Daniel unterstreicht, noch viel Raum für neue Perspektiven und Interpretationen. 
Funding Open Access funding provided by Projekt DEAL.

Open Access Dieser Artikel wird unter der Creative Commons Namensnennung 4.0 International Lizenz veröffentlicht, welche die Nutzung, Vervielfältigung, Bearbeitung, Verbreitung und Wiedergabe in jeglichem Medium und Format erlaubt, sofern Sie den/die ursprünglichen Autor(en) und die Quelle ordnungsgemäß nennen, einen Link zur Creative Commons Lizenz beifügen und angeben, ob Änderungen vorgenommen wurden.

Die in diesem Artikel enthaltenen Bilder und sonstiges Drittmaterial unterliegen ebenfalls der genannten Creative Commons Lizenz, sofern sich aus der Abbildungslegende nichts anderes ergibt. Sofern das betreffende Material nicht unter der genannten Creative Commons Lizenz steht und die betreffende Handlung nicht nach gesetzlichen Vorschriften erlaubt ist, ist für die oben aufgeführten Weiterverwendungen des Materials die Einwilligung des jeweiligen Rechteinhabers einzuholen.

Weitere Details zur Lizenz entnehmen Sie bitte der Lizenzinformation auf http://creativecommons.org/ licenses/by/4.0/deed.de.

\section{Bungert, Heike/Weiß, Jana (Hrsg.): ,God Bless America“. Zivilreligion in den USA im 20. Jahrhundert, 387 S., Campus, Frankfurt a. M./New York 2017.}

\section{Andreas Linsenmann}

Online publiziert: 19. Mai 2020

(C) Der/die Autor(en) 2020

Der Orientierungsbedarf im Hinblick auf Strukturmerkmale und Dynamiken der politischen Kultur der USA ist, obschon ohnehin konstant hoch, in den vergangenen Jahren nochmals gestiegen - getrieben nicht zuletzt durch das Bedürfnis, die Wahl Donald Trumps zum US-Präsidenten sowie deren Auswirkungen einordnen und verstehen zu können. Der vorliegende Sammelband, hervorgegangen aus einem Workshop des Centrums für Religion und Moderne und des Exzellenzclusters „Religion und Politik“ der Westfälischen Wilhelms-Universität Münster, bietet hierzu instruktive Ansätze. Er reiht sich ein in eine Neubefragung des für die dominanten Identitätskonstruktionen der USA wichtigen und seit den Anschlägen vom 11. September 2001 merklich revitalisierten Konzepts der Zivilreligion (civil religion). Dieser auf Jean-Jacques Rousseaus Hauptwerk „Le Contrat Social“ (1762) zurückgehende Ter-

\footnotetext{
A. Linsenmann $(\bowtie)$

Universität Koblenz-Landau, Koblenz, Deutschland

E-Mail: linsenmann@uni-koblenz.de
} 